\title{
Clinical factors affecting intraocular pressure change after orbital decompression surgery in thyroid-associated ophthalmopathy
}

This article was published in the following Dove Press journal:

Clinical Ophthalmology

18 January 2016

Number of times this article has been viewed

\author{
Jae Hoon Jeong' \\ Jeong Kyu Lee ${ }^{1,2}$ \\ Dong Ik Lee' \\ Yeoun Sook Chun' \\ Bo Youn $\mathrm{Cho}^{2}$ \\ 'Department of Ophthalmology, \\ College of Medicine, Chung-Ang \\ University ${ }^{2}$ Thyroid Center, Chung- \\ Ang University Hospital, Seoul, Korea
}

Objective: To report the physiological monitoring of intraocular pressure (IOP) during the postoperative periods after orbital decompression surgery and ascertain the correlation between the clinical factors and IOP changes.

Methods: The medical records of 113 orbits from 60 patients who underwent orbital decompression surgery were reviewed retrospectively. IOP measurement during the postoperative periods was classified based on the postoperative day: week 1 (1-7 days), month 1 ( $8-41$ days), month 2 (42-70 days), month 3 (71-97 days), month 4 (98-126 days), and final (after 127 days). The mean postoperative follow-up was 286.5 days for orbits with at least 6 months of follow-up. Univariate and multivariate linear regression analyses were performed to assess the correlation between the IOP reduction percentage and clinical factors.

Results: The mean IOP increased from 16.9 to $18.6 \mathrm{mmHg}(10.1 \%)$ at postoperative week 1 and decreased to $14.4 \mathrm{mmHg}(14.5 \%)$ after 2 months. Minimal little changes were observed postoperatively in the IOP after 2 months. Preoperative IOP had a significant positive effect on the reduction percentage both at postoperative week $1(\beta=2.51, P=0.001)$ and after 2 months $(\beta=1.07, P=0.029)$, and the spherical equivalent showed a positive correlation with the reduction level at postoperative week $1(\beta=1.71, P=0.021)$.

Conclusion: Surgical decompression caused a significant reduction in the IOP in thyroidassociated orbitopathy, and the amount of reduction was closely related to preoperative IOP; however, it may also cause a transient elevation in the IOP during the early postoperative phase in highly myopic eyes.

Keywords: Graves' ophthalmopathy, intraocular pressure, myopia, physiologic monitoring, postoperative periods, surgical decompression

\section{Introduction}

Thyroid-associated ophthalmopathy (TAO) causes multiple ophthalmic problems, including proptosis, diplopia, eyelid retractions, exposure keratitis, optic neuropathy, and cosmetic disfiguration. ${ }^{1}$ Further, elevation of intraocular pressure (IOP) is a well-known complication of TAO. Possible mechanisms for IOP elevation include secondary elevation of the episcleral venous pressure (EVP) by orbital congestion, ${ }^{2}$ increased retrobulbar pressure, ${ }^{3}$ restriction and compression of the globe by contraction of extraocular muscles, ${ }^{4,5}$ and mucopolysaccharide deposition in the trabecular meshwork. $^{6}$

Although, the main purpose of the orbital decompression is not to decrease IOP, previous studies have reported that IOP reduction may be caused due to orbital decompression surgery. ${ }^{7-11}$ Moreover, previous studies on the effect of orbital decompression surgery on IOP revealed IOP reduction after surgery; however, IOP increased within
Correspondence: Jeong Kyu Lee

Department of Ophthalmology,

Medicine, Chung-Ang University Hospital,

102 Heukseok-ro, Dongjak-gu, Seoul

06973, Korea

Tel +82262991688

Fax +8228251666

Email IkI246@gmail.com 
2 weeks after orbital decompression in our experience. In addition, the clinical factors that influence IOP change have not been researched, and limited literature is available.

The purpose of this study is to evaluate the change in IOP after orbital decompression surgery and to ascertain the clinical factors affecting IOP change. The results of this study can aid in predicting IOP changes after orbital decompression surgery in TAO patients in a clinical setting.

\section{Methods}

This study adhered to the tenets of the Declaration of Helsinki, and Institutional Review Board approval was obtained from the Chung-Ang University Hospital. Clinical charts of patients with TAO who underwent orbital decompression surgery from May 2010 to July 2013 at Chung-Ang University Hospital were reviewed. All patients who underwent decompression surgery over the study period were included in the study, but some patients were excluded based on the following criteria: incomplete preoperative or postoperative IOP data, previous history of ocular disease or ophthalmic surgery that could affect the IOP, and treatment with steroid pulse or orbital radiation therapy within 1 year before orbital decompression.

For each subject, clinical data collected from medical records included age, sex, smoking history, preoperative thyroidectomy or nonsurgical treatment history such as systemic steroid use and radiation therapy, pre- and postoperative degree of proptosis by Hertel exothalmometry, type of orbital decompression surgery, noncycloplegic refraction (Autorefractor KR-8900; Topcon Corporation, Tokyo, Japan), and IOP measurement by Goldmann applanation tonometer in the primary eye position.

\section{Intervention}

Patients with a stable thyroid function for at least 3 months underwent surgery under general anesthesia. Indications for surgery included disfiguring proptosis, severe exposure keratitis, and compressive optic neuropathy.

There were four types of surgical options: fat removal only and one to three-walled orbital decompression. The type of orbital decompression surgery was performed according to the patients' degree of proptosis. A medial or lateral wall was removed when a one-walled decompression surgery was planned, and if a two-walled decompression was planned, both walls were removed. A medial orbital wall approach was performed via a transcaruncular incision, and the ethmoidal bone was removed from the lacrimal crest to the orbital apex. A lateral wall approach was performed via upper eyelid skin crease incision, and the greater wing of the sphenoid bone was removed using a high-speed burr. Three-walled decompression surgery was performed by removing the medial and lateral wall plus the orbital floor via a transconjunctival approach while preserving the anterior inferomedial strut.

\section{Data analysis}

The IOP after orbital decompression surgery was classified into six categories according to the postoperative day as follows: week 1 (1-7 days), month 1 (8-41 days), month 2 (42-70 days), month 3 (71-97 days), month 4 (98-126 days), and final follow-up (after 127 days). The IOP was measured by taking the average of all the IOP recordings during the preoperative and subdivided postoperative periods in each subject's eyes. In addition, IOP measurement was terminated in subjects who underwent a surgical procedure that could affect the IOP during the follow-up period, such as strabismus surgery, eyelid surgery, and steroid injection into the eyelid. Percentage reduction of the IOP was calculated using the following formula:

$$
\frac{\text { Baseline IOP }- \text { Mean IOP during the period }}{\text { Baseline IOP }} \times 100
$$

Repeated-measures analysis of variance was used to compare the changes between the preoperative and postoperative IOP. Univariate analysis was used to assess the correlation between the percentage reduction in IOP and clinical factors. Multiple linear regression analysis, including variables with $P<0.2$ in the univariate analysis was performed to assess the effect of each variable on IOP reduction.

The probability level for statistical significance was set at 5\%. Data were recorded and analyzed using SPSS for Windows, version 20.0 (SPSS Inc., Chicago, IL, USA).

\section{Results}

Orbital decompression surgery was performed in 113 orbits from 60 patients with TAO, and the demographic and clinical characteristics of the subjects are presented in Table 1. The mean patient age was $30.5 \pm 7.9$ years (range: 17-54 years), and more than three-fourth (46/60, 76.7\%) of the patients were female. Nearly, all of the subjects underwent surgery in the inactive stage except three subjects with compressive optic neuropathy. The degree of proptosis was decreased to $5.15 \pm 1.64 \mathrm{~mm}$ after orbital decompression surgery $(P<0.001)$. Most of the subjects $(106 / 113,93.8 \%)$ underwent two- or three-walled surgical options, and the proportion was similar in two-wall and three-wall orbital decompression surgery. 
Table I Clinical and demographic characteristics of subjects with thyroid-associated orbitopathy who underwent orbital decompression surgery

\begin{tabular}{ll}
\hline Characteristics & Values \\
\hline Number of orbits & 113 \\
Age (years) & $30.5 \pm 7.9$ \\
Sex (male:female) & $25(22.1 \%): 88(77.9 \%)$ \\
Smoking & $36(31.9 \%)$ \\
Thyroidectomy & $19(16.8 \%)$ \\
Preoperative nonsurgical treatment (eyes) & \\
$\quad$ Systemic steroid use & $22(19.5 \%)$ \\
$\quad$ Systemic steroid with radiation therapy & $5(4.4 \%)$ \\
Degree of proptosis (mm) & \\
$\quad$ Preoperative & $20.19 \pm 1.81$ \\
$\quad$ Postoperative & $15.04 \pm 1.95$ \\
Type of orbital decompression surgery & \\
$\quad$ Fat removal only & $2(1.8 \%)$ \\
$\quad$ One-wall decompression & $5(4.4 \%)$ \\
$\quad$ Two-wall decompression & $51(45.1 \%)$ \\
$\quad$ Three-wall decompression & $55(48.7 \%)$ \\
Spherical equivalent (D) & $-3.24 \pm 3.15$ \\
Duration of follow-up (days) & $286.5 \pm 211.7$ \\
\hline
\end{tabular}

The serial changes in the IOP after orbital decompression surgery are shown in Table 2. The mean IOP was $16.89 \pm 4.10 \mathrm{mmHg}$ (range: $9.3-34.6 \mathrm{mmHg}$ ) before surgery, and it increased by $10 \%(18.59 \pm 3.68 \mathrm{mmHg}, P<0.001)$ at postoperative week 1 . This elevated IOP decreased to the preoperative status at postoperative month 1 , and it decreased consistently by $14 \%(14.44 \pm 3.71 \mathrm{mmHg}, P=0.008)$ of the preoperative IOP at postoperative month 2 . This significant reduction in IOP was maintained since after postoperative month 2, although the pressure changed at each interval during the follow-up period. For the duration of the follow-up period after postoperative month 2, the mean IOP was $14.55 \pm 4.17 \mathrm{mmHg}$ and the percentage reduction was $14.51 \% \pm 24.31 \%$.

Ten $(10 / 113,8.8 \%)$ orbits presented with high IOP $(>21 \mathrm{mmHg}$ ) before surgery and three of them had sustained high IOP after postoperative month 2, although it was lower than before the surgery. Although data were not included in the present study, three patients with IOP over $25 \mathrm{mmHg}$ were treated with eyedrops to lower the IOP before surgery; however, none of them needed continuous medical treatment for IOP reduction after the surgery. In contrast, IOP-lowering therapy was needed in one case after orbital decompression surgery, in spite of the fact that the IOP was within the normal range before the surgery. This was due to the spike in the IOP from 16.5 to $34.0 \mathrm{mmHg}$ at postoperative week 1, and it was normalized to $14 \mathrm{mmHg}$ at postoperative day 15 by temporary use of an antiglaucoma medication.

Tables 3 and 4 present the results of linear regression analysis of the correlation between the clinical factors and changes in IOP at postoperative week 1 and after postoperative month 2, respectively. Univariate linear regression analysis at postoperative week 1 demonstrated that three factors were significantly associated with the percentage reduction in IOP, that is, age ( $P=0.021)$, spherical equivalent $(P=0.044)$, and preoperative IOP $(P<0.001)$. In multivariate linear regression, spherical equivalent $(\beta=1.707, P=0.021)$ and preoperative IOP $(\beta=2.508, P=0.001)$ were also significantly associated with the percentage reduction in IOP after orbital decompression surgery at 1 week (Table 3 ).

In the analysis after postoperative month 2 , decrease in proptosis and IOP reduction percentage at week 1 were included for the long-term outcomes. In univariate linear regression, three factors were found to be significantly associated with the percentage reduction in IOP, that is, sex $(P=0.027)$, preoperative IOP $(P<0.001)$, and percentage reduction in IOP at week $1(P=0.024)$. In multivariate linear regression, only preoperative IOP was significantly ( $\beta=1.056, P=0.029$ ) associated with the percentage reduction in IOP (Table 4). The spherical equivalent showed significant association in the analysis at postoperative week 1 ; however, no significance was observed after postoperative month 2 .

Table 2 Serial change in the intraocular pressure after orbital decompression surgery

\begin{tabular}{|c|c|c|c|c|c|c|c|}
\hline Variables & Preoperative & Week I & Month I & Month 2 & Month 3 & Month 4 & Final follow-up \\
\hline Postoperative day & - & $\mathrm{I}-7$ & $8-41$ & $42-70$ & $7 I-97$ & $98-126$ & After 127 \\
\hline Average frequency of measurement & $3.15 \pm 2.20$ & $1.06 \pm 0.24$ & $|.08 \pm 0.3|$ & $1.07 \pm 0.26$ & $1.05 \pm 0.27$ & I. $13 \pm 0.42$ & $2.37 \pm 1.5 \mathrm{I}$ \\
\hline Mean IOP $(\mathrm{mmHg})$ & $16.89 \pm 4.10$ & $18.59 \pm 3.68$ & $16.46 \pm 3.49$ & $|4.44 \pm 3.7|$ & $14.46 \pm 3.47$ & $14.43 \pm 3.92$ & $|4.4| \pm 4.18$ \\
\hline Reduction in IOP $(\mathrm{mmHg})$ & - & $-1.7 I \pm 3.29$ & $0.42 \pm 2.60$ & $2.45 \pm 3.38$ & $2.44 \pm 3.61$ & $2.45 \pm 3.72$ & $2.48 \pm 3.15$ \\
\hline Percentage reduction in IOP (\%) & - & $-10.12 \pm 24.63$ & $2.54 \pm 15.78$ & $14.47 \pm 19.78$ & $14.34 \pm 18.76$ & $|4.5| \pm 24.28$ & $14.63 \pm 17.62$ \\
\hline$P$-value* & - & $<0.001$ & 0.112 & 0.008 & $0.009 *$ & 0.005 & $<0.001$ \\
\hline$P$-value** & - & - & $<0.001$ & 0.001 & 0.893 & 0.670 & 0.636 \\
\hline
\end{tabular}

Notes: *Comparison with preoperative intraocular pressure using the repeated-measures ANOVA with post hoc analysis. ***Comparison with the last follow-up using the repeated-measures ANOVA with post hoc analysis.

Abbreviations: ANOVA, analysis of variance; IOP, intraocular pressure. 
Table 3 Univariate and multivariate analyses of the association between clinical factors and change in the intraocular pressure at week I after orbital decompression surgery

\begin{tabular}{|c|c|c|c|c|c|c|}
\hline \multirow[t]{2}{*}{ Variables } & \multicolumn{3}{|c|}{ Univariate analysis } & \multicolumn{3}{|c|}{ Multivariate analysis } \\
\hline & $\beta$ coefficient & $\mathrm{Cl}(95 \%)$ & $P$-value & $\beta$ coefficient & $\mathrm{Cl}(95 \%)$ & $P$-value \\
\hline Age & 0.606 & 0.092 to 1.121 & 0.021 & 0.12 & -0.574 to 0.597 & 0.969 \\
\hline Sex & -9.200 & -20.247 to 1.848 & 0.102 & -2.757 & -13.250 to 7.735 & 0.603 \\
\hline Smoking & 4.581 & -5.606 to 14.767 & 0.374 & - & - & - \\
\hline Thyroidectomy & 7.486 & -6.023 to 20.995 & 0.274 & - & - & - \\
\hline Preoperative nonsurgical treatment & 0.231 & -4.751 to 5.213 & 0.927 & - & - & - \\
\hline Preoperative proptosis & 0.679 & -1.780 to 3.138 & 0.585 & - & - & - \\
\hline Type of orbital decompression surgery & 4.590 & -2.349 to $1 \mathrm{I} .529$ & 0.192 & 4.962 & -1.601 to 11.525 & 0.137 \\
\hline Spherical equivalent & 1.489 & 0.043 to 2.935 & 0.044 & 1.707 & 0.268 to 3.145 & 0.021 \\
\hline Preoperative intraocular pressure & 2.657 & 1.426 to 3.888 & $<0.00 \mathrm{I}$ & 2.508 & 1.052 to 3.964 & 0.001 \\
\hline
\end{tabular}

Abbreviation: $\mathrm{Cl}$, confidence interval.

Other factors, including age, sex, smoking history, preoperative thyroidectomy and nonsurgical treatment history, preoperative proptosis, and the type of orbital decompression surgery showed no significant association at postoperative week 1 and after postoperative month 2 .

\section{Discussion}

The current study was conducted to evaluate the change in IOP after orbital decompression surgery and the correlation between clinical factors and IOP change in patients with TAO. The authors demonstrated elevation in IOP (mean, $1.71 \mathrm{mmHg}, 10.1 \%$ ) during the initial postoperative phase (within 2 weeks) and reduction (mean $2.47 \mathrm{mmHg}, 14.5 \%$ ) after postoperative month 2 . The spherical equivalent and preoperative IOP were associated with the IOP reduction percentage during the initial phase; however, only preoperative IOP was found to affect IOP reduction after postoperative month 2 .
The results of IOP reduction postoperatively after 2 months, in the present study, corresponded well with those found in earlier clinical studies. ${ }^{7-10}$ However, there have been few studies on the change in IOP during the early postoperative phase. Robert et al ${ }^{11}$ showed a significant reduction in IOP from 19.3 to $17.0 \mathrm{mmHg}$ at 1 week after surgery and from 19.3 to $15.9 \mathrm{mmHg}$ at 3 months after surgery. Although the results of our study were similar during postoperative month 2, they were not consistent with the results of the early postoperative phase and with those reported by Robert et al, ${ }^{11}$ which may have been due to the type of orbital decompression surgery. In a majority of subjects $(93.8 \%)$ in this study, more than two orbital walls were removed; however, Robert et $\mathrm{al}^{11}$ performed only intraconal fat removal on 64 orbits.

A possible cause for this IOP elevation is the presence of inflammation and soft tissue swelling during the early postoperative period. The surgical procedure itself may induce inflammation and cause swelling of soft tissues

Table 4 Univariate and multivariate analyses of the association between the clinical factors and change in the intraocular pressure after postoperative 2 months

\begin{tabular}{|c|c|c|c|c|c|c|}
\hline \multirow[t]{2}{*}{ Variables } & \multicolumn{3}{|c|}{ Univariate analysis } & \multicolumn{3}{|c|}{ Multivariate analysis } \\
\hline & $\beta$ coefficient & $\mathrm{Cl}(95 \%)$ & $P$-value & $\beta$ coefficient & $\mathrm{Cl}(95 \%)$ & $P$-value \\
\hline Age & 0.070 & -0.283 to 0.423 & 0.695 & - & - & - \\
\hline Sex & -8.319 & -15.655 to -0.982 & 0.027 & -3.763 & -11.183 to 3.658 & 0.316 \\
\hline Smoking & 0.955 & -5.692 to 7.603 & 0.776 & - & - & - \\
\hline Thyroidectomy & 7.443 & -1.078 to 15.964 & 0.086 & 8.657 & -0.490 to 17.803 & 0.063 \\
\hline Preoperative nonsurgical treatment & -1.716 & -4.951 to 1.519 & 0.295 & - & - & - \\
\hline Preoperative proptosis & -0.528 & -2.204 to 1.147 & 0.533 & - & - & - \\
\hline Decrement of proptosis & 1.477 & -0.478 to 3.433 & 0.137 & 0.788 & -1.318 to 2.894 & 0.459 \\
\hline Type of orbital decompression surgery & 3.444 & -1.338 to 8.226 & 0.156 & 0.963 & -3.974 to 5.900 & 0.699 \\
\hline Spherical equivalent & -0.593 & -1.477 to 0.291 & 0.186 & -0.715 & -1.654 to 0.225 & 0.134 \\
\hline Preoperative intraocular pressure & 1.932 & 1.183 to 2.680 & $<0.00$ I & $\mathrm{I} .056$ & 0.112 to 2.001 & 0.029 \\
\hline IOP reduction percentage at week I & 0.161 & 0.021 to 0.300 & 0.024 & 0.093 & -0.061 to 0.247 & 0.235 \\
\hline
\end{tabular}

Abbreviations: $\mathrm{Cl}$, confidence interval; IOP, intraocular pressure. 
and extraocular muscles. Several previous studies ${ }^{12-14}$ have reported the expansion of the extraocular muscle volume following decompression surgery. Alsuhaibani et al ${ }^{14}$ found a significantly negative association between the time of postoperative computed tomography scans and change in the medial rectus muscle volume. They discussed that this may contribute to the lesser impact on the result during the early postoperative period. Therefore, as the postoperative expansion of extraocular muscle volume resolves, a marked IOP reduction may be observed after the acute inflammatory phase following orbital decompression surgery.

The preoperative IOP was the only factor that affected the amount of IOP reduction both during the early postoperative phase and after postoperative month 2 . Dev et $\mathrm{al}^{15}$ also reported that the degree of preoperative IOP elevation was found to be a strong predictor of the amount of IOP decrease after decompression by using binary categorical analysis, which was based on an IOP of $21 \mathrm{mmHg}$. To the best of our knowledge, this study represents the first linear regression analysis on IOP as a continuous variable in orbital decompression surgery. Other factors including age, sex, past medical history, proptosis, and type of orbital decompression surgery had no significant effect on the change in IOP after postoperative month 2 and this was similar to results from previous studies. . $^{8,10}$

A positive correlation between the preoperative IOP and reduction after decompression surgery could be described in terms of EVP. There was a study demonstrating the potential influence of hypothalamic centers on IOP, ${ }^{16}$ and an experimental study reported by Strohmaier et $\mathrm{a}^{17}$ suggested that the brainstem may play a role in controlling EVP. These reports indicated the presence of a central nervous system-controlled "set point" of EVP. Orbital decompression surgery increased the superior ophthalmic vein blood flow velocity by releasing the tension on the orbit and decreasing venous congestion. ${ }^{7,9}$ When congestion resolves after decompression surgery, the EVP falls to the individual's hypothalamic controlled set point, which leads to IOP reduction. Consequently, with a higher preoperative IOP, there is greater potential for IOP reduction.

Interestingly, the spherical equivalent showed significant positive association with the percentage reduction in IOP, with the exception of the preoperative IOP at initial postoperative phase, in linear regression analysis. This can be interpreted as the IOP during the early postoperative phase tends to increase more in myopic eyes, because an IOP increase is confirmed at 1 week after decompression surgery in the current study. The relation between myopia and postoperative IOP can be explained by the flexibility that is more prominent in myopic eyes. It has been suggested that hyperopic eyes demonstrated stiffer response parameters than myopic eyes, ${ }^{18}$ and pathological myopia might be related with the increased $\mathrm{IOP}^{19}$ in the studies using ocular response analyzer. The degree of myopia seems to affect postoperative IOP during the early inflammatory phase; however, it has had little influence beyond 2 weeks after surgery. In fact, the subject who was excluded from the present study by using antiglaucoma eyedrops after surgery due to a spike in the IOP had high myopia ( $-6.25 \mathrm{D})$ compared to the mean of the spherical equivalent $(-3.24 \mathrm{D})$ in total.

The present study has some limitations. The most decisive evidence supporting the change in IOP after the orbital decompression surgery would be a finding from a prospective case-controlled trial. However, this was a retrospective study, and we were unable to measure IOP at all the periods of the serial protocol in all subjects. Another limitation of this study is the generalization that the findings have high population-specific (Korean) aspects. Caucasian and Asian orbits and globe configurations have quantitative and qualitative differences ${ }^{20}$ that could affect IOP change in TAO. In addition, because we set follow-up visiting days instead of visiting time, there may exist diurnal variations in IOP measurement.

In conclusion, orbital decompression surgery in TAO could increase the IOP within 2 weeks of acute inflammatory postoperative phase; however, this transient IOP elevation reduces significantly compared to the preoperative level and stabilizes postoperatively after 2 months. The clinicians may need to consider the fact that orbital decompression surgery may significantly reduce the IOP in subjects with higher preoperative IOP; however, this can cause a prominent increase in the IOP during the early postoperative phase in high myopic eyes.

\section{Acknowledgments}

None of the authors have any financial or conflicting interests in any of the materials or methods mentioned in this article and have received any financial support related to this study. The authors received no government or nongovernment financial support for this study, and they have no proprietary or commercial interest in any of the methods or materials described in this article.

\section{Disclosure}

None of the authors had any competing interests in the present study. 


\section{References}

1. Woo KI, Kim YD, Lee SY. The clinical characteristics of thyroid orbitopathy in thyroid dysfunction patients in Korea. J Korean Ophthalmol Soc. 2008;49(9):1387-1396.

2. Kalmann R, Mourits MP. Prevalence and management of elevated intraocular pressure in patients with Graves' orbitopathy. Br JOphthalmol. 1998;82(7):754-757.

3. Otto AJ, Koornneef L, Mourits MP, Deen-van Leeuwen L. Retrobulbar pressures measured during surgical decompression of the orbit. Br J Ophthalmol. 1996;80(12):1042-1045.

4. Cockerham KP, Pal C, Jani B, Wolter A, Kennerdell JS. The prevalence and implications of ocular hypertension and glaucoma in thyroidassociated orbitopathy. Ophthalmology. 1997;104(6):914-917.

5. Fishman DR, Benes SC. Upgaze intraocular pressure changes and strabismus in Graves' ophthalmopathy. J Clin Neuroophthalmol. 1991;11(3): $162-165$.

6. Manor RS, Kurz O, Lewitus Z. Intraocular pressure in endocrinological patients with exophthalmos. Ophthalmologica. 1974;168(4): 241-252.

7. Danesh-Meyer HV, Savino PJ, Deramo V, Sergott RC, Smith AF. Intraocular pressure changes after treatment for Graves' orbitopathy. Ophthalmology. 2001;108(1):145-150.

8. Norris JH, Ross JJ, Kazim M, Selva D, Malhotra R. The effect of orbital decompression surgery on refraction and intraocular pressure in patients with thyroid orbitopathy. Eye (Lond). 2012;26(4):535-543.

9. Onaran Z, Konuk O, Oktar SO, Yucel C, Unal M. Intraocular pressure lowering effect of orbital decompression is related to increased venous outflow in Graves orbitopathy. Curr Eye Res. 2014;39(7):666-672.

10. Takahashi $Y$, Nakamura $Y$, Ichinose A, Kakizaki H. Intraocular pressure change with eye positions before and after orbital decompression for thyroid eye disease. Ophthal Plast Reconstr Surg. 2014;30(1):47-50.

11. Robert PY, Rivas M, Camezind P, Rulfi JY, Adenis JP. Decrease of intraocular pressure after fat-removal orbital decompression in Graves disease. Ophthal Plast Reconstr Surg. 2006;22(2):92-95.
12. Wenz R, Levine MR, Putterman A, Bersani T, Feldman K. Extraocular muscle enlargement after orbital decompression for Graves' ophthalmopathy. Ophthal Plast Reconstr Surg. 1994;10(1):34-41.

13. Hu WD, Annunziata CC, Chokthaweesak W, et al. Radiographic analysis of extraocular muscle volumetric changes in thyroid-related orbitopathy following orbital decompression. Ophthal Plast Reconstr Surg. 2010;26(1):1-6.

14. Alsuhaibani AH, Carter KD, Policeni B, Nerad JA. Effect of orbital bony decompression for Graves' orbitopathy on the volume of extraocular muscles. Br J Ophthalmol. 2011;95(9):1255-1258.

15. Dev S, Damji KF, DeBacker CM, Cox TA, Dutton JJ, Allingham RR. Decrease in intraocular pressure after orbital decompression for thyroid orbitopathy. Can J Ophthalmol. 1998;33(6):314-319.

16. Samuels BC, Hammes NM, Johnson PL, Shekhar A, McKinnon SJ, Allingham RR. Dorsomedial/Perifornical hypothalamic stimulation increases intraocular pressure, intracranial pressure, and the translaminar pressure gradient. Invest Ophthalmol Vis Sci. 2012;53(11):7328-7335.

17. Strohmaier CA, Reitsamer HA, Kiel JW. Episcleral venous pressure and IOP responses to central electrical stimulation in the rat. Invest Ophthalmol Vis Sci. 2013;54(10):6860-6866.

18. Roberts CJ, Reinstein DZ, Archer TJ, Mahmoud AM, Gobbe M, Lee L. Comparison of ocular biomechanical response parameters in myopic and hyperopic eyes using dynamic bidirectional applanation analysis. J Cataract Refract Surg. 2014;40(6):929-936.

19. Oner V, Tas M, Ozkaya E, Oruc Y. Effect of pathological myopia on biomechanical properties: a study by ocular response analyzer. Int J Ophthalmol. 2015;8(2):365-368.

20. Eckstein LA, Shadpour JM, Menghani R, Goldberg RA. The relationship of the globe to the orbital rim. Arch Facial Plast Surg. 2011;13(1): $51-56$.
Clinical Ophthalmology

\section{Publish your work in this journal}

Clinical Ophthalmology is an international, peer-reviewed journal covering all subspecialties within ophthalmology. Key topics include: Optometry; Visual science; Pharmacology and drug therapy in eye diseases; Basic Sciences; Primary and Secondary eye care; Patient Safety and Quality of Care Improvements. This journal is indexed on Submit your manuscript here: http://www.dovepress.com/clinical-ophthalmology-journal

\section{Dovepress}

PubMed Central and CAS, and is the official journal of The Society of Clinical Ophthalmology (SCO). The manuscript management system is completely online and includes a very quick and fair peer-review system, which is all easy to use. Visit http://www.dovepress.com/ testimonials.php to read real quotes from published authors. 